Documenta \& Instrumenta

ISSN-e: 1697-3798

http://dx.doi.org/10.5209/DOCU.64058

\title{
La serie RRC 441 NERI Q. VRB, una emisión de la ciudad de Roma
}

\author{
Luis Amela Valverde ${ }^{1}$
}

Recibido: 25 de abril de 2018 / Aceptado: 3 de abril de 2019

Resumen. La serie monetal RRC 441/1 fue emitida a inicios del año 49 a.C. cuando había ya estallado la guerra civil entre C. Julio César (cos. I 59 a.C.) y el Senado. Significado y lugar de acuñación. Palabras clave. Guerra Civil; Cn. Nerio; iconografía; Roma; Apolonia.

\section{[en] The Series RRC 441 NERI q. VRB, An Issue of the City of Rome}

\begin{abstract}
The monetary series RRC 441/1 was issued at the beginning of the year 49 B.C. When civil war broke out between C. Julius Caesar (cos. I 59 BC) and the Senate. Meaning and place of minting. Keywords. Second Civil War; Cn. Nerio; iconography; Rome; Apollonia.
\end{abstract}

Cómo citar: L Amela Valverde, "La serie RRC 441 NERI Q. VRB, una emisión de la ciudad de Roma”, Documenta \& Instrumenta, 17 (2019), pp. 11-17. 
La serie RRC 441/1 es una emisión de denarios efectuada en la ciudad de Roma a inicios del año 49 a.C. ${ }^{2}$. Su cronología está asegurada por la mención de los dos cónsules de ese año en el reverso: L. Cornelio Léntulo Crus y C. Claudio Marcelo (el Mayor) $^{3}$, a los que encontraremos mencionados en otra emisión (RRC 445/1-3).

$\mathrm{Su}$ descripción es la siguiente:

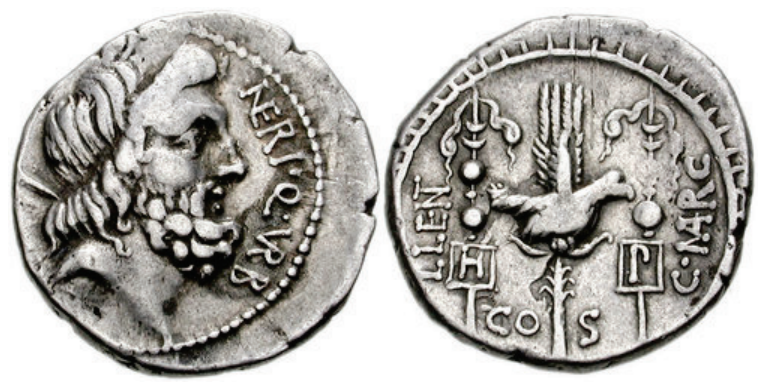

RRC 441/1 = HGC 3 19. Denario ${ }^{4}$.

Anv.: cabeza de Saturno a dra., detrás harpa sobre el hombro; delante, $\mathrm{NERI} \cdot \mathrm{Q} \cdot \mathrm{VRB}$ hacia abajo. Gráfila de puntos.

Rev.: águila legionaria entre dos estandartes manipulares, uno con $\mathrm{H}$ (hastati) a izq. y otro con P (principes) a dra; a izq. L·LENT hacia arriba; a dra., C·MARC hacia arriba; debajo CO-S.

Cuños se anverso: $[<20]$. Cuños de reverso: $[<22]^{5}$.

Se mencionan la existencia de un denario que no incluye la palabra VRB, del cual diversos autores dudan de su existencia, por lo que no lo incluyen en su catálogo 6 .

Si bien aparentemente contemporánea de la acuñación de Q. Sicinio (RRC 440/1), fue una emisión excepcional producida a nombre de $\mathrm{Cn}$. Nerio, quien fue quaestor urbanus en el momento del estallido de la guerra civil ${ }^{7}$. La fecha consular de la emisión es inusual ${ }^{8}$, al aparece por primera vez en la numismática ${ }^{9}$. Posible-

2 CRAWFORD, Roman Republican Coinage, p. 89. K. Kopij, Auctoritas et dignitas: the study of propaganda in the period of the Late Roman Republic on the example of the Pompey family (gens Pompeia Magna) in the light of archaeological and written sources, Kraków, 2017, p. 116.

3 Classical Numismatic Group, Inc. (CNG), Electronic Auction 157, lote no 211, de 31 de enero de 2007.

4 Crawford, Roman Republican Coinage, p. 460.

5 SEABY, Roman Silver Coins..., p. 69. CALICÓ Y CALICÓ, Los denarios romanos anteriores a J.C..., p. 201. La web especializada https://numismatica-classica.lamoneta.it/moneta/R-G92/1 tampoco lista tal variedad.

6 SEABY, Roman Silver Coins..., p. 69. CALICÓ Y CALICÓ, Los denarios romanos anteriores a J.C..., p. 201. La web especializada https://numismatica-classica.lamoneta.it/moneta/R-G92/1 tampoco lista tal variedad.

7 SEAR, The History and Coinage of the Roman Imperatores..., p. 5.

8 BABELON, Description Historique et Chronologique... vol. I, p. 350; Description Historique et Chronologique... vol. II, p. 253. GRUEBER, Coins of the Roman Republic in the British Museum. Vol. I, p. 504 n. 2. CRAWFORD, Roman Republican Coinage, p. 461. SEAR, Roman Coins and their Values..., p. 151. CATALLI, La monetazione romana repubblicana, p. 241. B. WOYTEK, B. (2003): Arma et Nummi. Forschungen zur römischen Finanzgeschichte und Münzprägung der Jahre 49 bis 42 v. Chr., Wien., 2003, p. 97; "The Denarii RRC 453/3, signed by the consuls Lentulus and Marcellus (49 BC): A Die Study", en Neue 
mente, sería una forma de legitimar la emisión de moneda ${ }^{10}$, ya cuando el conflicto con de C. Julio César (cos. I 59 a.C.) había estallado.

La obvia naturaleza de carácter militar de esta acuñación ${ }^{11}$ indicaría que se inició después de que se recibiera en Roma la noticia del cruce del Rubicón por parte de César ${ }^{12}$, dando origen a la segunda guerra civil romana (49-45 a.C.). Quizás incluso esta acuñación pudiera interpretarse como una respuesta a los famosos denarios del elefante de César (RRC 443/1) ${ }^{13}$. En ese momento no podía haber ninguna duda de que la rivalidad entre César y el Senado tendría que resolverse por la fuerza de las armas, y estos tipos, por lo tanto, habrían sido más apropiados para la ocasión ${ }^{14}$.

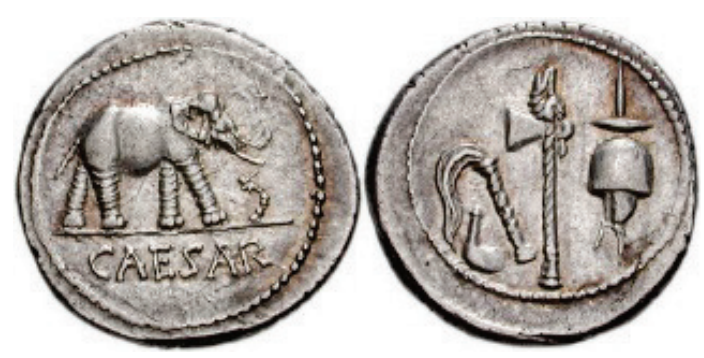

Denario de César RRC 443/1, llamado “del elefante" por motivos obvios ${ }^{15}$.

Como sabemos que el Senado propuso otorgar fondos del Erario a Pompeyo (Caes. BCiv. 1, 6, 3. Dio Cass. 41,3,4), quizás la presente acuñación sea la respuesta a tal moción ${ }^{16}$, como indican las insignias militares del reverso ${ }^{17}$, que aluden, evidentemente, al ejército ${ }^{18}$, en alusión al futuro que se avecina ${ }^{19}$.

Muy interesante es la aportación de K. Kopij. Para este autor, las representaciones del anverso y reverso pueden formar un todo único. En el aerarium populi Romani, que estaba en el Templo de Saturno en el Foro Romano, en el tiempo de la paz, tradicionalmente se almacenaban estandartes legionarios. Por lo tanto, esta acuñación puede simbolizar la extracción de los signos militares del tesoro y el

Forschungen zur Münzprägung der Römischen Republik - Beiträge zum internationalen Kolloquium im Residenzschloss Dresden, München, 2016, 173-214, p. 175.

9 DE FRANCISCO, La datación por magistrados..., p. 145 n. 193.

10 BABELON, Description Historique et Chronologique... vol. II, p. 253. GRUEBER, Coins of the Roman Republic in the British Museum. Vol. I, p. 504 n. 2. WOYTEK, Arma et Nummi..., p. 100.

11 WOYTEK, Arma et Nummi..., p. 98; “The Denarii RRC 453/3...”, p. 175.

12 AMISANO, La storia di Roma antica e le sue monete..., p. 154.

13 NOUSEK, "Turning Points in Roman History...", p. 305. Sobre la serie RRC 443/1 vid: L. AMELA VALVERDE, "La emisión cesariana RRC 443/1 y sus imitaciones", Numisma 246 (2002), 7-37; "De nuevo sobre el denario de César con elefante (RRC 443/1)", Minerva 26 (2013), 145-162.

14 SEAR, The History and Coinage of the Roman Imperatores..., p. 5.

15 CLASSICAL Numismatic Group, Inc. (CNG), no 812854 (https://cngcoins.com/Coin.aspx?CoinID=124578).

16 BATTENBERG, Pompeius und Caesar..., p. 77.

17 BATTENBERG, Pompeius und Caesar..., p. 78.

18 BABELON, Description Historique et Chronologique... vol. I, p. 350; Description Historique et Chronologique... vol. II, p. 253. GRUEBER, Coins of the Roman Republic in the British Museum. Vol. I, p. 504 n. 2. SEABY, Roman Silver Coins..., p. 69. CRAWFORD, Roman Republican Coinage, p. 461.

19 SCHMITT Y PRIEUR, Les monnaies romaines, p. 131. 
comienzo de la guerra, o la esperanza de paz y su reubicación en el Templo de Saturno $^{20}$.

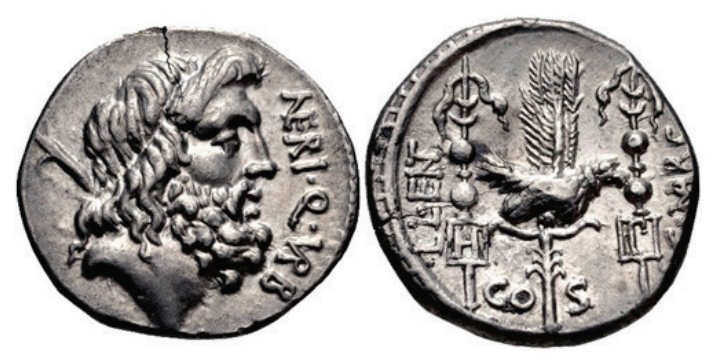

Denario RRC 441/1 (ampliado x 2) ${ }^{21}$.

A destacar que el reverso de la emisión de Nerio es una imitación del denario efectuado por C. Valerio Flaco (cos. 93 a.C.) en Massalia (Marsella) durante el año 82 a.C. (RRC 365/1) (Ra $^{22}$ Laparición de estandartes en relación con los hastati y los príncipes, los cuales, junto con los triarii, formaban el ejército romano anterior a las reformas de éste por C. Mario (cos. I 107 a.C.), parece ser un anacronismo ${ }^{23}$, fruto del mimetismo en copiar el reverso del original de Flaco o, de otro modo, una forma de atraer a filas a la gente mediante un recordatorio bien conocido por parte de la población.
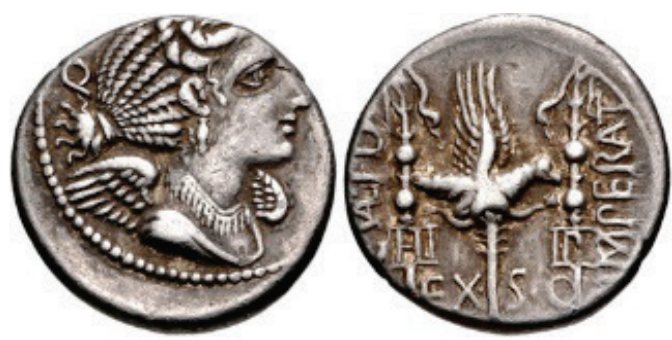

Denario de C. Valerio Flaco (RRC 365/1b) ${ }^{24}$.

El cruce del Rubicón por parte de César (App. BCiv. 2, 34. Caes. BCiv. 1, 7-8. Suet. Caes. 31) posiblemente significó la finalización de la acuñación RRC 440/1 $\mathrm{y}$, por el contrario, el inicio de la presente, que debió verse interrumpida por la aparición de las fuerzas cesarianas en Roma. Con su retirada a Grecia, el bando

20 KOPIJ, Auctoritas et dignitas..., p. 117.

21 CLASSICAL NUMISMATIC GROUP. INC. (CNG), Electronic Auction 237, lote n⿳ 328, de 21 de julio de 2010.

22 WOYTEK, Arma et Nummi..., p. 98; “The Denarii RRC 453/3...”, p. 175. KOPIJ, Auctoritas et dignitas..., p. 117. Sobre esta amonedación, vid: A. ALFÖLDI, "Les deniers de C. Valerius Flaccus freppés à Marseille et les dernières émissions de drachmes massaliotes", $R N 1$ (1969), 55-61.

23 Y. LE BOHEC, "Les légendes H et P sur le monnaies aux enseignes de 82 et 49 avant Jésus-Christ", Recherches \& Travaux 54 (1998), 35-42 p. 58 ofrece una nueva lectura de las letras H y P, y considera que en realidad figura Honos y Pax o Pietas, aunque su interpretación no ha tenido fortuna.

24 Classical Numismatic Group, Inc. (CNG), Electronic Auction 297, lote n 306, de 27 de febrero de 2013. 
senatorial cedió el control de la producción de monedas de la capital. Todas las posteriores emisiones fueron producidas por establecimientos temporales que suministraban dinero a medida que la situación militar requería. La Casa de la Moneda de Roma se convirtió así pues en una herramienta de César y de su facción y, a partir de entonces, permaneció firmemente en manos de César, incluso después del asesinato del Dictador en el año 44 a.C. ${ }^{25}$

La cabeza de Saturno (como indica la presencia del harpa) alude al hecho de que $\mathrm{Cn}$. Nerio era cuestor urbano ${ }^{26}$. Tal motivo indica que la amonedación fue acuñada por orden del Senado, ya que su templo, en el Foro romano, era la sede del Erario.

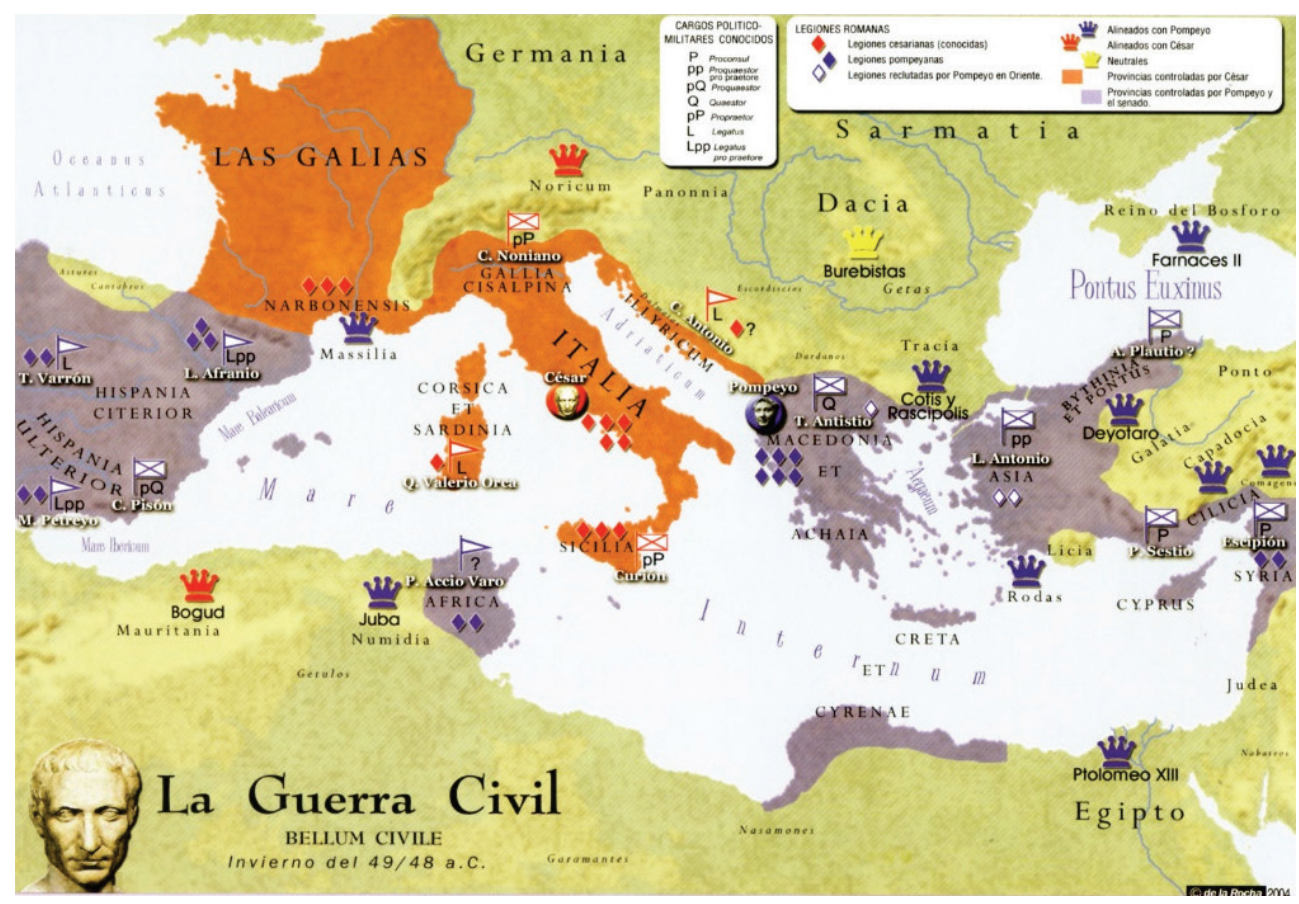

Situación de las fuerzas militares en el invierno del año 49/48 a.C., según Carlos de la Rocha. En realidad, la situación descrita pertenecería más bien a finales del mes de marzo del año 49 a.C., momento en que César ha ocupado Italia y las islas de Sicilia, Córcega y Cerdeña.

Si bien parece claro que la mención de que Nerio era cuestor urbano no deja lugar a dudas de que fue emitida en la ciudad de $\operatorname{Roma}^{27}$ (en caso contrario, no ten-

25 SEAR, The History and Coinage of the Roman Imperatores..., p. 5.

26 BABELON, Description Historique et Chronologique... vol. I, p. 350; Description Historique et Chronologique... vol. II, p. 253. GRUEBER, Coins of the Roman Republic in the British Museum. Vol. I, p. 504 n. 2. SEABY, Roman Silver Coins..., p. 60. CRAWFORD, Roman Republican Coinage, p. 461. BATTENBERG, Pompeius und Caesar..., p. 77. CATALLI, La monetazione romana repubblicana, p. 241. SCHMITT Y PRIEUR, Les monnaies romaines, p. 131.

27 AMISANO, La storia di Roma antica e le sue monete..., p. 154. 
dría sentido tal alusión) ${ }^{28}$, se ha considerado otros lugares donde se habría producido esta emisión, como: la isla de Sicilia ${ }^{29}$ o en Iliria (quizás en la ciudad de Apolonia) ${ }^{30}$. Esta última localización ha cogido últimamente fuerza debido a su defensa por B. Woytek, quien considera que debido a cuestiones técnicas y al hallazgo en 1963 de un tesoro ${ }^{31}$ en un suburbio de Tirana (la capital de Albania) conteniendo cinco ejemplares de esta serie (junto con piezas RRC 445/1a-b, que se han atribuido precisamente a Apolonia) ${ }^{32}$, refuerza su teoría de que esta serie habría sido acuñada en Iliria, en donde se encontraban los cónsules, que podían haber evidentemente supervisado esta acuñación de carácter militar ${ }^{33}$. A ello habría que sumar la ausencia de nuestra emisión en el tesoro de Brandosa (RRCH 352), que cierra precisamente con la emisión de Q. Sicinio (RRC 440/1) $)^{34}$, la última acuñación regular de la ceca de Roma ${ }^{35}$.
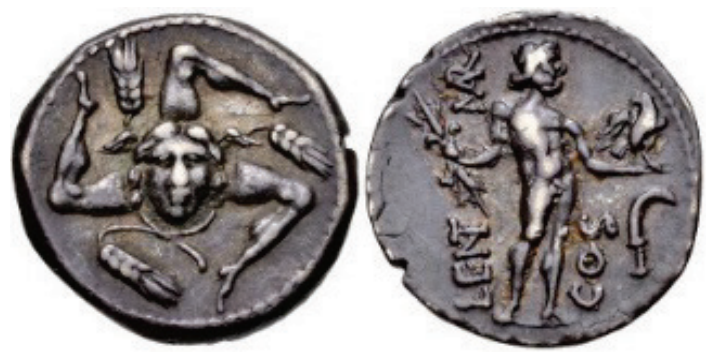

Denario RRC $445 / 1 \mathrm{a}^{36}$.

28 M. H. CRAWFORD, “Bernhard Woytek. Arma et Nummi...”, Gnomon 84 (2012), 337-342, p. 339.

29 COHEN, Description générale de les monnaies..., pp. 111 y 228.

30 BABELON, Description Historique et Chronologique... vol. II, p. 253. WOYTEK, Arma et Nummi..., pp. 100 y 558; 2016, 175. O. D. HOOVER, The Handbook of Greek Coinage Series, Volume 3. Handbook of Macedon and Its Neighbors. Part I: Macedonia, Illyria, and Epeiros. Sixth to First Centuries BC, Lancaster/London, 2016, pp. 5 y 10. R. ALBERT, Die Münzen der Römischen Republik. Von der Anfängen bis zur Schlacht von Actium (4. Jahrhundert v. Chr. Bis 31 v. Chr.), Regenstauf, $2011^{2}$, p. 193 duda entre esta atribución y Roma.

31 Este ocultamiento está compuesto por 24 dracmas ilirios de Apolonia y Dirraquio más 39 denarios romanorepublicanos.

32 BABELON, Description Historique et Chronologique... vol. II, p. 253. GRUEBER, Coins of the Roman Republic in the British Museum. Vol. II, p. 467 n. 1. CRAWFORD, Roman Republican Coinage, pp. 89 y 462. Battenberg, Pompeius und Caesar..., p. 80. A. BURNETT, M. AMANDRY Y P. P. RIPOLLÈS, Roman Provincial Coinage. Volume I. From the death of Caesar to the death of Vitellius (44 B.C.-A.D. 69). Part I: Introduction and Catalogue, London, 1992, pp. 287-288. SEAR, The History and Coinage of the Roman Imperatores..., p. 7. WOYTEK, Arma et Nummi..., p. 555. HOOVER, The Handbook of Greek Coinage Series..., pp. 5 y 10. H. ZEHNACKER, "Rupture ou continuité: La monnaie romaine de Sylla à Auguste", $B A G B$ (1990), 175-189, p. 182. K. KOPIJ, "The Coins Related to Pompey the Great through the Lens of the Theology of Victory", en Proceedings of the $6^{\text {th }}$ International Numismatic Congress in Croatia Rijeka, 2011, 141150, p. 149. AMISANO, La storia di Roma antica e le sue monete..., pp. 155-156.

33 WOYTEK, Arma et Nummi..., p. 100; “The Denarii RRC 453/3...,", p. 175. Como señala M. W. METCALF, "Bernhard Woytek. Arma et Nummi...", SNR 85 (2016), 222-230, p. 224, es muy sugerente que el hasta de RRC 445/1a pueda estar relacionado con el que figura junto a la cabeza de Saturno de la emisión de Nerio (RRC 441/1) que aquí presentamos.

34 WOYTEK, Arma et Nummi..., p. 100.

35 BATtenBERG, Pompeius und Caesar..., p. 76. SEAR, The History and Coinage of the Roman Imperatores..., p. 4. WOYTEK, Arma et Nummi..., p. 96.

36 Classical Numismatic Group, Inc. (CNG), Electronic Auction 366, lote n⿳ 740, de 13 de enero de 2016. 
Ciertamente, como indica B. Woytek, Nerio no tuvo mucho tiempo para producir moneda antes de la llegada de las fuerzas cesarianas, ya que quizás su fabricación se iniciaría a partir del día 7 de enero al otorgar poderes especiales a Pompeyo a través de un senatus consultum ultimum (App. BCiv. 2, 31. Dio Cass. 40, 64, 4. Oros. 6, 15, 1. Plut. Ant. 5, 2; Pomp. 59, 1) y sólo dispondría de diez días para ello, por no decir que la ceca estaría en plena efervescencia con la amonedación de Sicinio (RRC 440/1) ${ }^{37}$. Así mismo, habría que aludir a un detalle técnico: en vez de presentar una grafila de puntos, común a las emisiones efectuadas en la ciudad de Roma durante este periodo, exhibe una línea sólida, que iría pues en contra de la atribución tradicional de esta amonedación ${ }^{38}$.

Pero, repetimos, no tendría sentido entonces mencionar la magistratura completa de Nerio, así como el súbito parón que sufre esta acuñación, en teoría debido a la presencia de las fuerzas de César cerca de Roma, que obligó a evacuar la Urbs a la facción senatorial. Como señala con cierto humor H. Zehnacker, no tendría por qué extrañar que los optimates en fuga se llevaran algunos sacos de monedas, que darían origen a las piezas aparecidas en el citado ocultamiento ${ }^{39}$. Igualmente, no se entendería que los cónsules supervisaran la emisión de Nerio cuando ellos mismos estarían efectuando su propia amonedación en Apolonia (RRC 445/1).

En definitiva, hay que concluir que la emisión de Nerio (RRC 441/1) fue efectuada en la ciudad de Roma, sea inmediatamente después, o, de manera simultánea, con la serie de Q. Sicinio (RRC 440/1), o, como ya insinuó M. H. Grueber, esta amonedación se inició en la ciudad de Roma y tras el avance cesariano fue efectuada en una ceca móvil por Italia ${ }^{40}$. Sea como fuere, nosotros preferimos la primera solución.

WOYTEK, Arma et Nummi..., p. 98.

WOYTEK, Arma et Nummi..., p. 99; “The Denarii RRC 453/3...”, p. 175.

H. ZEHNACKER, "Bernhard Woytek. Arma et Nummi...”, RN 161 (2005), 227-230, p. 229.

40 WOYTEK, Arma et Nummi..., p. 100 no descartaba la posibilidad de que esta emisión se produjera en el sur de Italia, durante la retirada optimate. 